\title{
Endothelial Nitric Oxide Synthase (eNOS) S1176 phosphorylation status governs atherosclerotic lesion formation
}

Running Title: eNOS S1176 in atherosclerosis

\author{
Monica Y. Lee ${ }^{1 *^{*}}$, Nur-Taz Rahman ${ }^{2}$, William C. Sessa ${ }^{1}$ \\ ${ }^{1}$ Department of Pharmacology, Vascular Biology and Therapeutics Program, Yale \\ University School of Medicine, New Haven, CT, 06520 USA \\ ${ }^{2}$ Bioinformatics Support Group, Yale University Cushing/Whitney Medical Library, New \\ Haven, CT, 06520 USA \\ $¥$ Present address: Department of Physiology and Biophysics, Center for Cardiovascular \\ Research, University of Illinois at Chicago School of Medicine, Chicago, IL, 60612 USA
}

*Corresponding author: Monica Y. Lee, Ph.D., Center for Cardiovascular Research, University of Illinois at Chicago School of Medicine, College of Medicine Research Building 835 S. Wolcott Ave., Chicago, IL 60612. Tel: 312-355-3176 Fax: 312-996-1414 Email: MonicaYL@uic.edu

Total Word Count (including Title Page, Abstract, Text, References, Tables and Figures Legends): 6561 


\section{ABSTRACT (Word Count: 246)}

Objective: We have previously demonstrated the in vivo importance of the Akt-eNOS substratekinase relationship, as defective postnatal angiogenesis characteristic of global Akt1-null mice is rescued when bred to 'gain-of-function' eNOS S1176D mutant mice. While multiple studies support the cardioprotective role of endothelial NO generation, the causal role of Akt1-dependent eNOS S1176 phosphorylation during atherosclerotic plaque formation is not yet clear.

Approach \& Results: We herein bred congenic 'loss-of-function' eNOS S1176A and 'gain-offunction' eNOS S1176D mutant mice to the proatherogenic Akt1 ${ }^{--} ;$ApoE $^{-/-}$double knockout mice to definitively test the importance of Akt-mediated eNOS S1176 phosphorylation during atherogenesis. We find that a single amino acid substitution at the eNOS S1176 phosphorylation site yields divergent effects on atherosclerotic plaque formation, as an eNOS phospho-mimic aspartate (D) substitution at S1176 leads to decreased indices of atherosclerosis, even when on a proatherogenic Akt1 global deletion background. Conversely, mice harboring an unphosphorylatable mutation to alanine (S1176A) result in increased lipid deposition and cellular apoptosis, phenocopying the physiological consequence of eNOS deletion and/or impaired enzyme function. Furthermore, gene expression analyses of whole aortas indicate a combinatorial detriment from NO deficiency and Western Diet challenge, as 'loss-of-function' eNOS SA mice on a high-fat and high-cholesterol diet present a unique expression pattern indicative of augmented T-cell activity when compared to eNOS S1176D mice.

Conclusions: By using genetic epistasis approaches, we conclusively demonstrate that Aktmediated eNOS S1176 phosphorylation and subsequent activation remains to be the most physiologically relevant method of NO production to promote cardioprotective effects. 


\section{INTRODUCTION}

Endothelial cells (EC) play a vital role in modulating vascular responses, as endothelial dysfunction and reduced nitric oxide (NO) bioavailability are common features underlying the pathophysiological risks for insulin resistance, hypertension, and atherogenic dyslipidemia ${ }^{1,2}$. The importance of NO production in cardiovascular protection has been long-established through investigation of numerous physiological models. Endothelial production of NO is critical for the regulation of several vascular responses, including vascular tone, blood flow, leukocyteendothelial interactions, platelet adhesion/aggregation, and vascular smooth muscle cell function. A decrease in NO bioavailability results in endothelial dysfunction, as characterized by features conducive to the development of atherosclerosis to influence parameters such as thrombosis, inflammation, neointimal proliferation, and vasoconstriction ${ }^{3,4}$.

$\mathrm{NO}$ is generated through conversion of L-arginine to L-citrulline by the enzymatic action of nitric oxide synthase (NOS), an NAPDH-dependent NO synthase. This reaction requires calcium/calmodulin, FAD, FMN, and tetrahydrobiopterin (BH4) as necessary cofactors for efficient NO production. Various studies have been conducted to understand the contribution of eNOS activity on vascular protection through manipulation of essential cofactors required for eNOS enzymatic activity or through modulation of eNOS protein levels and expression ${ }^{5,6}$. eNOS knockout mice demonstrate the most extreme outcomes of eNOS loss, such as increased platelet aggregation, increased leukocyte-endothelial interaction, and increased vascular smooth muscle cell proliferation ${ }^{5,7}$. Furthermore, eNOS knockout mice manifest whole-body outcomes indicative of consequent NO deficiency, such as hypertension, insulin resistance, and hyperlipidemia phenotypes that are directly correlated with accelerated atheroma formation upon Western diet challenge ${ }^{5}$. Overexpression of eNOS also, paradoxically, yields larger atherosclerotic lesions, which was later attributed to consequential eNOS dysfunction and uncoupling ${ }^{6}$. eNOS uncoupling is therefore an important mechanism underlying the pathogenesis of EC dysfunction and downstream atherogenesis, where increasing evidence suggests that proper functionality of the eNOS enzyme, rather than expression levels, is most critical for cardiovascular homeostasis ${ }^{8}$.

Endothelial NOS (eNOS) enzymatic activity can be regulated on several levels in addition to cofactor availability. These include gene transcription/mRNA stability, interactions with key modulators (i.e. hsp90, caveolin), and through post-translational modifications (PTM). eNOS can undergo various PTMs to incur an effect on function and hence influence NO production, including S-nitrosylation, acetylation or glutathionlyation. However, a large body of research since its initial discovery in the 1990s has focused on how phosphorylation status at specific residues affect eNOS function ${ }^{9,10}$. Among the various mechanisms of regulation, eNOS protein phosphorylation at S1176 (1179 in bovine, 1177 in human and 1176 in murine) plays a critical role in promoting activation of eNOS enzymatic activity ${ }^{11,12}$. Various extracellular stimuli (e.g. insulin, VEGF, shear stress) generate NO through eNOS S1176 phosphorylation, where several kinases (e.g. Akt, PKA, PKG, AMPK, CaMKII, etc.) have been identified, implying the physiological importance of eNOS phosphorylation and activation ${ }^{2}$.

Although several kinases can phosphorylate eNOS at S1176, previous studies using genetic epistasis approaches demonstrate that the kinase Akt is the most critical for eNOS phosphorylation and function in vivo. We have shown that the impaired angiogenic phenotypes typically seen in Akt1 deficient mice are rescued when crossed with eNOS 'gain-of-function' (S1176D) mice ${ }^{12}$. The observed rescue in impaired angiogenesis demonstrates the importance 
of Akt1 activity as the predominant kinase for eNOS phosphorylation in vascular repair. While the Akt-eNOS relationship has been described in the context of adaptive angiogenesis, the causal role of Akt-dependent eNOS phosphorylation in atherogenesis has not yet been investigated using the available mouse models. The definitive role of the Akt1 signaling pathway in atherosclerosis remains uncertain, as several in vitro studies suggest Akt may serve either an athero-protective or athero-prone role ${ }^{13-15}$. Moreover, the contradicting results using eNOS overexpression systems emphasizes the importance of proper eNOS function, rather than overt modulation of expression and protein levels ${ }^{5,8}$.

In this study, we aimed to investigate the Akt1-eNOS activation cascade on atherosclerotic plaque development using various genetically modified mice. Knock-in mice carrying the described 'gain-of-function' (S1176D) or a 'loss-of-function' (S1176A) mutations in the endogenous eNOS gene were bred to $\mathrm{Akt1}^{---} ; \mathrm{ApoE}^{--}$double knockout mice ${ }^{16}$ to investigate the definitive role of Akt-mediated eNOS signaling in atherogenesis. We herein report that a single amino acid substitution at the eNOS S1176 phosphorylation site yields divergent effects on atherosclerotic plaque formation when placed on an $\mathrm{Akt1}^{---}$; $\mathrm{ApoE}^{-- \text {double }}$ donockout genetic background. Our study provides evidence that the preservation of eNOS function is essential to mitigate atherosclerotic lesion formation, despite the global loss of Akt1 expression. Moreover, gene expression analyses indicate that inadequate NO production together with a Western Diet challenge promotes vascular signatures reflective of the adaptive immune response. We herein demonstrate that Akt1-directed eNOS activation indeed serves a vasculo-protective role, where we further substantiate the Akt1-eNOS axis as the major signaling mechanism that links endothelial integrity to cardiovascular disease outcome. 


\section{METHODS}

\section{Animal Procedures}

Mice expressing an endogenous phosphomimetic (S1176D) and unphosphorylatable (SA) eNOS point-mutations at the S1176 site were backcrossed to a C57BI6 background (provided by Paul L. Huang ${ }^{12,17}$ ). Akt1 ${ }^{--}$; ApoE ${ }^{-1-}$ double knockout mice were previously backcrossed onto a C57BI6 background and utilized for the studies herein ${ }^{16}$. These triple allele mice hence reflect eight generations of backcrossing to a C57BI/6 background. Previous studies indicate the importance of Akt1 expression and eNOS function for proper Mendelian ratios ${ }^{18,19}$. Akt1 heterozygous females (SA [or S1176D $]^{+++} ; \mathrm{Akt1}^{+-} ; \mathrm{ApoE}^{--_{-}}$) were therefore bred to Akt1 homozygote KO males (SA [or S1176D] ${ }^{+++} ; \mathrm{Akt1}^{---} ; \mathrm{ApoE}^{-I_{-}}$) to maximize the chances of viable offspring. Although genetic crossing strategies were employed, the global eNOS S1176A mutation on a sub-optimal Akt1 expression system resulted in significantly decreased litter and offspring numbers (average $\mathrm{n}=3$ pups per litter, unpublished). Furthermore, our studies were limited to male offspring harboring homozygote triple-allelic mutations (SA [or S1176D] ${ }^{+/+}$; Akt1 $^{-/-}$; $\mathrm{ApoE}^{-{ }^{-}}$) to minimize variability and additionally maintain the difficult genetic lines, hence the low numbers in these studies.

Triple allelic homozygote mice were maintained on standard chow, where at $\sim 8 w k s$ of age mice were fed ad libitum with a high-fat (40\% kcal), high-cholesterol (1.25\%) Western Diet feed (Research Diets, D12108). Adult mice were fed with Western Diet for $\sim 4$ or 12 weeks for atherosclerosis studies. All experiments were approved by the Institutional Animal Care Use Committee at Yale University.

\section{Oil Red $O$ en face staining}

After a 12-week Western Diet challenge, mice were anesthetized (ket/xyl) and perfused with PBS ( $\mathrm{pH} 7.4)$ prior to removal of the aorta. Aortas were dissected from the aortic valve to the iliac bifurcation and fixed in $4 \%$ PFA/PBS ( $\mathrm{pH} 7.4)$ overnight at $4^{\circ} \mathrm{C}$. Aorta samples were thoroughly cleaned prior to longitudinal opening and pinning down in silicone plates for en face preparation. Lipid-rich lesions were identified using previously described Oil Red $\mathrm{O}$ staining techniques ${ }^{16}$. Images were acquired using a Nikon SMZ 1000 microscope and lesion areas were blindly quantified using ImageJ software. Lesion areas are reported as percentages of total aortic area. Data reflect multiple cohorts of mice.

\section{Immunostaining}

Mice were anesthetized (ket/xyl) and perfused with PBS $(\mathrm{pH} 7.4)$ prior to removal of target organs (e.g. brachiocephalic artery, heart). Samples were fixed in $4 \%$ PFA/PBS overnight at $4^{\circ} \mathrm{C}$ followed by immediate dehydration in $30 \%$ sucrose/PBS for OCT embedding. Frozen tissues were cut at 5um sections and stained with hematoxylin/eosin or Masson's Trichrome. Adjacent sections were immunostained with the following antibodies: anti-CD68 (Serotec, MCA1957); anti-VCAM1 (BD 550547). Cell apoptosis was measured using an in situ cell death detection kit and following the manufacturer protocol (Roche, 12156792910). For all parameters, multiple sections were assessed for each tissue sample where the average values are reported for each mouse. Data reflect multiple cohorts of mice.

\section{Gene Expression Analyses}


Upon euthanasia (ket/xly), mice were perfused with PBS and aortic tissue (ascending aorta to the femoral arteries) was quickly isolated. Whole aorta samples were thoroughly cleaned of perivascular fat and immediately flash-frozen in liquid nitrogen to minimize technical effects imposed by time (within 20 minutes of euthanasia). Whole aorta tissues were then processed for RNA extraction and quality checks. RNA quality was assessed using spectrophotometric (NanoDrop) readings to ensure that all samples were at A260/280 ratios of $\sim 2.0$. Satisfactory samples were prepared for hybridization reactions with the NanoString platform (PanCancer Immune Profile Mouse). In brief, the NanoString nCounter Gene Expression panels allows for the analyses of up to $\sim 800$ RNA targets selected for published significance in key biological pathways using a digital detection and direct molecular barcoding approach. Normalized expression counts were extracted from the NanoString nSolver software for further data analyses using Qlucore Omics Explorer® (New York, USA). Samples were assigned into their respective groups: SA+CHOW (2 samples), S1176D+CHOW (3 samples), SA+WD (3 samples), and S1176D+WD (3 samples), and the 783 genes in the dataset were used to generate PCA plots and heatmaps. Best clustering of the samples was achieved with multi-group comparison of all 4 groups at a pvalue $\leq 0.01$ (corresponding q-value $\leq 0.084$ ), which identified 91 differentially expressed genes (Supplemental Figure 2). These genes and their subsets were used to generate heatmaps, and both genes and the groups were subject to hierarchical clustering. Gene ontology and gene set enrichment analyses were carried out using these different gene sets using Ingenuity Pathway Analysis (IPA, Qiagen, MD). From IPA we obtained predicted activation/inhibition of canonical pathways, predicted upstream regulators, and illustration of mechanistic pathways.

\section{Statistics}

All data are shown as mean \pm standard error of the mean (SEM). Statistical significance was evaluated using a Student's $t$ test. Significance was determined based on a $p$ value calculation of $p<0.05$. 


\section{RESULTS}

eNOS-S1176A phospho-impaired mutant mice display exacerbated aortic lesion formation upon 12wk-Western Diet challenge.

Mice expressing an endogenous phosphomimetic (S1176D) and unphosphorylatable (SA) eNOS point-mutation at S1176 were crossed with $\mathrm{Akt1}^{-- \text {; }} \mathrm{ApoE}^{-- \text {double }}$ knockout mice to generate triple allelic homozygote mice. Introduction of these mutations do not affect the levels of eNOS protein levels, but rather modulates eNOS activity through manipulation of the S1176 site. Triple allelic homozygote mice at $\sim 8$ weeks of age were fed a Western Diet for a duration of 12 weeks, where manipulation of eNOS had no effect on total weight, irrespective of the diet type (Figure 1A). However, en face Oil Red O staining of aortas from eNOS S1176A mice show larger areas of aortic lesions when compared to eNOS S1176D mice (Figure 1B \& 1C). Lesion quantifications show significantly increased plaque areas in eNOS S1176A mice throughout regions of the aortic arch (Figure 1D), thoracic aorta (Figure 1E), and abdominal aorta (Figure 1F).

\section{Atherosclerotic mice expressing 'gain-of-function' eNOS mutant (S1176D) exhibit less plaque necrosis apoptosis and inflammation.}

Cross-sectional analyses of the aortic sinus indicate that 'gain-of-function' eNOS S1176D mice develop visibly decreased atherosclerotic lesions and lipid deposition when compared to eNOS S1176A mice (Figures 2A \& 2B), corroborating the decreased plaque formation seen throughout the whole aorta. Expression of the 'gain-of-function' eNOS S1176D mutant also results in reduced fibrosis with decreased necrotic core regions, as shown by Masson's Trichrome staining (Figures 2C \& 2D). We additionally examined cellular apoptosis using terminal deoxynucelotidyl transferase dUTP end-labeling staining techniques in adjacent cross sections of the aortic sinus, where eNOS SA mutant mice exhibit a significant increase in overall TUNEL+ plaque regions (Figures 2E \&2F). Cross-sectional analyses of the brachiocephalic artery also indicate significantly larger plaques in the eNOS SA mice along with increased lipid deposition and apoptosis (Supp. Fig. 1). These results suggest that the eNOS SA 'loss-of-function' mice exhibit suboptimal levels of endothelial-derived NO prior to the onset of Western Diet feeding that contribute to exacerbated plaque formation. Next, we examined the infiltration of macrophages and VCAM-1 levels in the two strains. The greater lesion size in eNOS S1176A mice is accompanied by enhanced CD68 positive cells in the plaque and VCAM-1 levels, where these effects are reduced in eNOS S1176D mice (Figure 3A \& 3B, quantified in 3C \& 3D). The increase in inflammatory cell and VCAM1 expression corroborates previously reported increases of VCAM1 in Akt1-null mice, where enhanced proinflammatory gene expression was found to be secondary to macrophage infiltration ${ }^{16}$. Expression of the 'gain-of-function' eNOS S1176D mutation exhibits decreased indices of inflammation despite the lack of Akt1 expression and chronic environmental stress of Western Diet challenge. These findings support the long-held notion that increasing NO bioavailability mitigates plaque formation, emphasizing the cardioprotective role of eNOS function.

Previous studies have shown that endothelial adhesion molecule expression and subsequent monocyte recruitment/proliferation requires at least 2 weeks of a hypercholesterolemic environment ${ }^{20}$. Moreover, adherent monocytes are found on the surface of activated endothelium in the thoracic aorta prior to the development of foam cell-rich fatty streaks 
within 4-weeks of Western Diet challenge in ApoE mice ${ }^{21}$. We therefore shortened the Western Diet feed duration to a 4-week duration to reflect early endothelial activation, and comprehensively profiled the transcriptional changes in whole aorta samples between comparison groups to capture the various mechanisms of atherogenesis. Importantly, we also isolated the whole aorta to account for the long-range cardioprotective effects of $\mathrm{NO}$ as a gaseous agent on the various cells of the vasculature. Samples were then processed on a NanoString Immune Profile platform to determine how eNOS dysfunction together with a 4-week Western Diet challenge affects aortic gene expression, with focus on adaptive and innate immune response genes. A heatmap of relative expression data illustrating unsupervised hierarchical gene clustering between the comparison groups show an array of genetic clusters across the groups of interest (Figure 4A, $\mathrm{p} \leq 0.01,91$ genes). Gene expression counts were further analyzed using Ingenuity Pathway Analysis (IPA) software to identify canonical pathways and upstream regulators associated with the genetic changes reflective of eNOS functionality and dietary condition. Multigroup comparison yields a subset of genes significantly associated with 'Cardiac Hypertrophy', likely reflecting the known effects of impaired eNOS function on cardiomyocyte function ${ }^{22}$. We further identified a large subset of genes associated with 'Hepatic Fibrosis Signaling', 'Neuroinflammation Signaling', and 'Dendritic Cell Maturation' pathways (Figure 4B). The genetic changes associated with Western Diet and impaired eNOS function also yield a signature where lipopolysaccharide, TNF, dexamethasone, and STAT3 were identified as upstream regulators (Figure 4C). The enhanced lesion formation in eNOS S1176A mice upon Western Diet challenge underscores the importance of proper eNOS function and resultant NO levels to maintain an athero-protective environment.

\section{Gene expression profile comparison between the eNOS mutant groups on Western Diet reveal $T$ cell activation.}

Atherosclerosis is a chronic inflammatory disease of the vessel wall driven by both innate and adaptive immune responses ${ }^{23}$. While lesion development is largely driven by monocyte infiltration and subendothelial retention, plaque progression also relies on adaptive immune cells, as autoimmunity against ApoB involves CD4+ T-helper cells ${ }^{24}$. We further analyzed the dataset to identify genetic changes associated with eNOS phosphorylation state by directly comparing the eNOS mutant groups. A heatmap of relative expression data illustrate that most inflammatory genes are upregulated in the 'loss-of-function' eNOS S1176A mice (Figure 5A, p $\leq 0.01,28$ genes). Interestingly, pathway analyses identify that upon Western Diet challenge, a single point mutation affecting eNOS function results in preferential changes to regulate Th1/Th2 activation and STAT3 signaling pathways (Figure 5B). Along with previously identified regulators, IPA also determined IL-1b as an upstream activator. IL-1b is a well-known local and systemic contributor to cardiovascular inflammation, where the recent CANTOS clinical trial outcomes affirm IL-1b as an atherosclerosis-relevant inflammatory target. T-cell receptors (TCR) and CD28, one of the most effective T-cell co-stimulatory receptors, were also identified as exclusive upstream regulators under these comparison conditions (Figure 5C). The emergent genetic signatures reveal an association of NO deficiency to an early activation of T cell-associated pathways as a potential mechanism for atherosclerotic disease progression.

Gene expression analyses identify a unique set of differentially regulated genes in eNOS S1176A on a Western Diet. 
Our analyses indicate that inadequate NO production together with high-fat/highcholesterol diet leads to inflammatory vascular signatures engaging the adaptive immune response. The eNOS S1176A mutant mice exhibit a subset of genes that are differentially upregulated and unique to the combined effect of both 'loss-of-function' eNOS SA mutation and a Western Diet feeding period of 4 weeks (Figure 4A). We therefore preferentially queried for genes differentially upregulated only in the eNOS S1176A mice fed a WD compared to all other comparison groups to better understand the combinatorial effects of NO deficiency and Western Diet ( $p \leq 0.05,59$ genes Figure 6A). Further analyses of the identified 59 genes using IPA yielded several upregulated pathways, including 'Role of PKR in Interferon induction and Antiviral Response', 'Regulation of the EMT by Growth Factors, and 'Coronavirus Pathogenesis' (Figure 6B). The cluster of enriched genes is predicted to be the result of several key inflammatory regulators, namely through activation of IFNy and TNF pathways (Figure 6C). Pathways analyses also implicate NFkB/RelA activation in the eNOS S1176A + WD group, and is likely engaged for downstream transcriptional effects of heightened IFNy and $\mathrm{TNF}^{25}$. Additional upstream regulators identified in this analyses include IL-2, where the major source of IL-2 is derived from CD4+ and CD8+ T-cells ${ }^{26}$. The lack of bioavailable NO levels may therefore promote atherogenesis through coordinated activation of inflammatory pathways selective for $\mathrm{T}$ cells and adaptive immunity. These themes may also be implicated in the emerging vascular-related pathogenic mechanisms of coronaviruses.

\section{Tumor Necrosis Factor (TNF) and Interferon-gamma (IFNY) cytokines are likely elevated in atherosclerotic mice expressing the 'loss-of-function' eNOS mutation (S1176A).}

Pathway analyses repeatedly identify TNF and IFNy as two inflammatory and immune-activation related cytokines as upstream activators of the differentially expressed gene signature unique to the combinatorial effects of diminished NO (eNOS S1176A) and Western Diet challenge (Figures 4B, 5B, and 6B). We therefore generated the predicted pathways via IPA and superimposed our gene expression data onto the molecular pathway components (Figure 7). As shown, many of the genes in the pathway that are upregulated in eNOS S1176A mice fed a WD are predicted to be regulated by an activated TNF or IFNy, suggesting that these two cytokines are likely responsible for the increased atherogenesis. More importantly, this study clarifies the existing discrepancy in the field surrounding eNOS, where our results affirm that eNOS expression and proper function is necessary to maintain an atheroprotective environment. 


\section{DISCUSSION}

The present study provides conclusive evidence through genetic epistasis approaches the importance of the Akt-eNOS kinase-substrate relationship for proper eNOS enzymatic function and cardiovascular benefit. Analysis at anatomical regions predisposed for atherosclerotic plaque formation indicate that mice harboring an unphosphorylatable (SA) eNOS point-mutation at the S1176 site results in increased lipid deposition, cellular apoptosis, and indices of inflammation, where pathway analyses identify preferential engagement of $\mathrm{T}$ cell activation pathways. These deleterious effects are drastically reduced in the phospho-mimetic eNOS S1176D mice, suggesting a direct and significant correlation between $\mathrm{NO}$ bioavailability and atherogenic capacity. Through genetic modification of the eNOS S1176 activation site, we show that Akt1mediated eNOS phosphorylation is critical for potentiating the vasculoprotective effects of endothelial Akt-eNOS signaling when challenged with dietary conditions that promote atherosclerosis.

Published studies show that both diabetes mellites and hypercholesterolemia synergistically promote the formation of complex atherosclerotic lesions that are associated with attenuated vascular Akt signaling ${ }^{27}$. Additionally, hypercholesterolemia promotes impaired endothelial-dependent vasorelaxation, where decreased NO bioavailability is an early characteristic of atherosclerosis ${ }^{28}$. While supplementation with eNOS substrates and cofactors enhance eNOS-derived NO production, an imbalance in eNOS enzymatic activity and cofactor levels can lead to eNOS uncoupling and detrimental effects ${ }^{29,30}$. Furthermore, mice overexpressing eNOS paradoxically show increased atherosclerotic lesion formation, emphasizing the complexity of eNOS regulation ${ }^{8}$. We therefore addressed the importance of Aktmediated eNOS S1176 phosphorylation in atherogenesis through site-specific modulation of enzymatic activity rather than eNOS protein levels, where 'loss-of-function' eNOS S1176A indeed display worsened atherosclerosis. Recent studies also show increased atherosclerosis in eNOS SA mice when on an ApoE background, similar to eNOS/ApoE DKO mice ${ }^{31}$. However, these studies were performed in the presence of intact Akt expression and does not directly compare outcomes to the phospho-mimetic eNOS S1176D mutation. Our genetic comparison therefore definitively shows that eNOS S1176 phosphorylation-dependent NO generation is sufficient to decrease atherosclerotic lesion progression, even in the absence of Akt1 expression. The delayed atherosclerotic plaque formation observed in eNOS S1176D mice reinforces eNOS S1176 phosphorylation as a therapeutic target for ameliorating vascular pathologies associated with endothelial dysfunction.

Mice lacking both eNOS and ApoE also display increased blood pressure when compared to ApoE-null mice ${ }^{32}$, suggesting that accelerated atherosclerosis may instead be due to systemic hypertension. However, treatment of eNOS/ApoE DKO with hydralazine, an ACE-independent vasodilator, resulted in lowered blood pressure with no effect on atherosclerotic lesion areas ${ }^{33}$. Furthermore, ApoE KO mice treated with levels of L-NAME low enough to maintain normotension also exhibit endothelial dysfunction and increased atherosclerotic lesions ${ }^{34}$. These studies suggest that NO deficiency may promote atherosclerosis through mechanisms independent of blood pressure regulation. Whereas endothelial targeted deletion of Akt1 results in spontaneous hypertension, global deletion of Akt1 does not affect blood pressure, likely due to the loss of Akt1 in other major cell types necessary for vascular tone (i.e smooth muscle cells). The use of a global Akt1-null background together with the endogenous mutation of eNOS S1176 serves as a unique 
model, where we herein identify a genetic signature that may elucidate the blood pressureindependent consequences of eNOS deficiency.

Atherosclerosis is often regarded as a chronic response to arterial tissue inflammation engaging both innate and adaptive immunity ${ }^{23}$. Evidence implicating the adaptive immune system has grown considerably in the last couple decades, as hypercholesterolemia can elicit a heighted activation state of T-cells. Moreover, atherosclerotic plaques are primarily associated with CD4+ T-cells characterized by heightened IFNy production ${ }^{23}$. Hence, immune responses mediated by the adaptive immune system may be the dominant force to promote inflammation in mature atherosclerotic lesions. Our pathways analyses indicate that the eNOS activation state modulates T-cell activity, as NO deficiency together with a Western Diet challenge leads to a combinatorial increase in select genes that are predicted to be a result of increased TNF and IFNy levels. Our gene expression data also identify 'Regulation of the EMT Transition by Growth Factor Pathways' as a top canonical pathway implicated under conditions of decreased NO bioavailability and Western Diet feeding. Elegant lineage-tracing studies have recently demonstrated that endothelial-to-mesenchymal (endoMT) transitioning occurs during atherosclerosis, where inflammatory conditions (i.e TNF + IFNy) were shown to further promote endoMT processes ${ }^{35,36}$. Suboptimal levels of endothelial-derived NO predispose the large vasculature to an inflammatory state where exposure to a high-fat/high-cholesterol diet promotes the preferential activation of TNF and interferon-related pathways that may contribute to the recently described occurrences of endoMT in atherogenesis. This will, however, require further investigation.

eNOS activation has been shown to also occur through modification of alternative sites. Previous studies have shown potential mechanisms of eNOS activation independent of S1176 phosphorylation $^{31}$. Recent studies show that insulin may promote endothelin receptor B (EDNRB) expression and NO production in ECs for alternate eNOS-S1176 independent mechanisms. These studies, however, utilized overexpression systems for endothelial overexpression ( $2-3$ fold) of IRS1 to achieve enhanced insulin signaling. Moreover, while their studies demonstrate a potential for eNOS-S1176 independent NO generation mechanisms, the proposed mechanism for increased endothelial EDNRB expression is entirely reliant on intact PI3K/Akt signaling. Our model system herein investigates the effect of eNOS S1176 point-mutation on an atherogenic Akt1-null global deletion background, where the genetic loss of Akt1 expression therefore prevents the potential for any protective effect mediated through Akt signaling. While eNOS S1176-independent mechanisms of NO production exist, our results suggest that Akt-mediated eNOS S1176 phosphorylation remains to be the most physiologically relevant method of eNOS activation and subsequent NO production. This study therefore shows not only the importance of eNOS S1176 phosphorylation and subsequent NO production, but the relevance of intact Akt signaling, further reinforcing the physiological importance of the Akt1-eNOS kinase-substrate relationship for endothelial and vascular health. 


\section{ACKNOWLEDGEMENTS}

We thank Paul L. Huang for providing the mutant eNOS mice for these studies. This work was supported in part by grants from the National Institutes of Health (grants R35 HL139945 and P01 HL1070205 to W.C.S, R00 HL130581 to M.Y.L.).

\section{AUTHOR CONTRIBUTIONS}

M.Y.L. generated and characterized the mouse phenotypes. N.R. performed the gene expression analyses and GSEA. M.Y.L. designed the project and experimental approaches. M.Y.L and W.C.S. wrote the manuscript, which was reviewed and edited by all authors. 


\section{REFERENCES}

1. Huang PL. eNOS, metabolic syndrome and cardiovascular disease. Trends Endocrinol Metab. 2009;20(6):295-302. doi:10.1016/j.tem.2009.03.005

2. Garcia V, Sessa WC. Endothelial NOS: perspective and recent developments. $\mathrm{Br} \mathrm{J}$ Pharmacol. 2019;176(2):189-196. doi:10.1111/bph.14522

3. Liu VWT, Huang PL. Cardiovascular roles of nitric oxide: A review of insights from nitric oxide synthase gene disrupted mice†. Cardiovasc Res. 2008;77(1):19-29. doi:10.1016/j.cardiores.2007.06.024

4. Huang PL. Mouse Models of Nitric Oxide Synthase Deficiency. J Am Soc Nephrol. Published online 2000:4.

5. Kuhlencordt PJ, Gyurko R, Han F, et al. Accelerated Atherosclerosis, Aortic Aneurysm Formation, and Ischemic Heart Disease in Apolipoprotein E/Endothelial Nitric Oxide Synthase Double-Knockout Mice. Circulation. 2001;104(4):448-454. doi:10.1161/hc2901.091399

6. Ozaki M, Kawashima S, Yamashita T, et al. Overexpression of endothelial nitric oxide synthase accelerates atherosclerotic lesion formation in apoE-deficient mice. J Clin Invest. 2002;110(3):331-340. doi:10.1172/JCI0215215

7. Moroi M, Zhang L, Yasuda T, et al. Interaction of genetic deficiency of endothelial nitric oxide, gender, and pregnancy in vascular response to injury in mice. J Clin Invest. 1998;101(6):1225-1232. doi:10.1172/JCI1293

8. Kawashima S, Yokoyama M. Dysfunction of Endothelial Nitric Oxide Synthase and Atherosclerosis. Arterioscler Thromb Vasc Biol. 2004;24(6):998-1005. doi:10.1161/01.ATV.0000125114.88079.96

9. Fulton D, Gratton JP, McCabe TJ, et al. Regulation of endothelium-derived nitric oxide production by the protein kinase Akt. Nature. 1999;399(6736):597-601. doi:10.1038/21218

10. Dimmeler S, Fleming I, Fisslthaler B, Hermann C, Busse R, Zeiher AM. Activation of nitric oxide synthase in endothelial cells by Akt-dependent phosphorylation. Nature. 1999;399(6736):601-605. doi:10.1038/21224

11. Atochin DN, Huang PL. Endothelial nitric oxide synthase transgenic models of endothelial dysfunction. Pflüg Arch - Eur J Physiol. 2010;460(6):965-974. doi:10.1007/s00424-0100867-4

12. Schleicher M, Yu J, Murata T, et al. The Akt1-eNOS Axis Illustrates the Specificity of Kinase-Substrate Relationships in Vivo. Sci Signal. 2009;2(82):ra41-ra41. doi:10.1126/scisignal.2000343

13. Rotllan N, Chamorro-Jorganes A, Araldi E, et al. Hematopoietic Akt2 deficiency attenuates the progression of atherosclerosis. FASEB J. 2015;29(2):597-610. doi:10.1096/fj.14-262097 
14. Arranz A, Doxaki C, Vergadi E, et al. Akt1 and Akt2 protein kinases differentially contribute to macrophage polarization. Proc Natl Acad Sci. 2012;109(24):9517-9522.

doi:10.1073/pnas.1119038109

15. Kerr BA, Ma L, West XZ, et al. Interference with Akt Signaling Protects Against Myocardial Infarction and Death by Limiting the Consequences of Oxidative Stress. Sci Signal.

2013;6(287):ra67-ra67. doi:10.1126/scisignal.2003948

16. Fernández-Hernando C, Ackah E, Yu J, et al. Loss of Akt1 Leads to Severe Atherosclerosis and Occlusive Coronary Artery Disease. Cell Metab. 2007;6(6):446-457.

doi:10.1016/j.cmet.2007.10.007

17. Kashiwagi S, Atochin DN, Li Q, et al. eNOS phosphorylation on serine 1176 affects insulin sensitivity and adiposity. Biochem Biophys Res Commun. 2013;431(2):284-290. doi:10.1016/j.bbrc.2012.12.110

18. Cho H, Thorvaldsen JL, Chu Q, Feng F, Birnbaum MJ. Akt1/PKBa Is Required for Normal Growth but Dispensable for Maintenance of Glucose Homeostasis in Mice. J Biol Chem. 2001;276(42):38349-38352. doi:10.1074/jbc.C100462200

19. Shesely EG, Maeda N, Kim HS, et al. Elevated blood pressures in mice lacking endothelial nitric oxide synthase. Proc Natl Acad Sci. 1996;93(23):13176-13181.

doi:10.1073/pnas.93.23.13176

20. Zhu SN, Chen M, Jongstra-Bilen J, Cybulsky MI. GM-CSF regulates intimal cell proliferation in nascent atherosclerotic lesions. J Exp Med. 2009;206(10):2141-2149.

doi:10.1084/jem.20090866

21. Yuqing Huo, C. L. Ramos, K. Ley. Rolling and adhesion of monocytes to early atherosclerotic lesions of apolipoprotein E-/-(apoE-/-) mice requires P-selectin, PSGL-1, /spl alpha//sub 4/ integrin and VCAM-1. In: Proceedings of the First Joint BMES/EMBS Conference. 1999 IEEE Engineering in Medicine and Biology 21st Annual Conference and the 1999 Annual Fall Meeting of the Biomedical Engineering Society (Cat. N. Vol 1. ; 1999:56 vol.1. doi:10.1109/IEMBS.1999.802082

22. Yang Xiao-Ping, Liu Yun-He, Shesely Edward G., Bulagannawar Manohar, Liu Fang, Carretero Oscar A. Endothelial Nitric Oxide Gene Knockout Mice. Hypertension. 1999;34(1):24-30. doi:10.1161/01.HYP.34.1.24

23. Wolf D, Ley K. Immunity and Inflammation in Atherosclerosis. Circ Res. 2019;124(2):315327. doi:10.1161/CIRCRESAHA.118.313591

24. Kimura Takayuki, Kobiyama Kouji, Winkels Holger, et al. Regulatory CD4+ T Cells Recognize Major Histocompatibility Complex Class II Molecule-Restricted Peptide Epitopes of Apolipoprotein B. Circulation. 2018;138(11):1130-1143. doi:10.1161/CIRCULATIONAHA.117.031420

25. Pfeffer LM. The Role of Nuclear Factor кB in the Interferon Response. J Interferon Cytokine Res. 2011;31(7):553-559. doi:10.1089/jir.2011.0028 
26. Liao W, Lin JX, Leonard WJ. IL-2 family cytokines: new insights into the complex roles of IL2 as a broad regulator of T helper cell differentiation. Curr Opin Immunol. 2011;23(5):598604. doi:10.1016/j.coi.2011.08.003

27. Hamamdzic D, Fenning RS, Patel D, et al. Akt pathway is hypoactivated by synergistic actions of diabetes mellitus and hypercholesterolemia resulting in advanced coronary artery disease. Am J Physiol-Heart Circ Physiol. 2010;299(3):H699-H706.

doi:10.1152/ajpheart.00071.2010

28. Steinberg Helmut O., Bayazeed Basel, Hook Ginger, Johnson Ann, Cronin Jessica, Baron Alain D. Endothelial Dysfunction Is Associated With Cholesterol Levels in the High Normal Range in Humans. Circulation. 1997;96(10):3287-3293. doi:10.1161/01.CIR.96.10.3287

29. Hattori Y, Hattori S, Wang X, Satoh H, Nakanishi N, Kasai K. Oral Administration of Tetrahydrobiopterin Slows the Progression of Atherosclerosis in Apolipoprotein E-Knockout Mice. Arterioscler Thromb Vasc Biol. 2007;27(4):865-870. doi:10.1161/01.ATV.0000258946.55438.0e

30. Cosentino F, Katusic ZS. Tetrahydrobiopterin and Dysfunction of Endothelial Nitric Oxide Synthase in Coronary Arteries. Circulation. 1995;91(1):139-144. doi:10.1161/01.CIR.91.1.139

31. Park K, Mima A, Li Q, et al. Insulin decreases atherosclerosis by inducing endothelin receptor B expression. JCI Insight. 2016;1(6). doi:10.1172/jci.insight.86574

32. Knowles JW, Reddick RL, Jennette JC, Shesely EG, Smithies O, Maeda N. Enhanced atherosclerosis and kidney dysfunction in eNOS-I-Apoe-/- mice are ameliorated by enalapril treatment. J Clin Invest. 2000;105(4):451-458. doi:10.1172/JCI8376

33. Chen J, Kuhlencordt PJ, Astern J, Gyurko R, Huang PL. Hypertension Does Not Account for the Accelerated Atherosclerosis and Development of Aneurysms in Male Apolipoprotein E/Endothelial Nitric Oxide Synthase Double Knockout Mice. Circulation. 2001;104(20):23912394. doi:10.1161/hc4501.099729

34. Kauser K, da Cunha V, Fitch R, Mallari C, Rubanyi GM. Role of endogenous nitric oxide in progression of atherosclerosis in apolipoprotein E-deficient mice. Am J Physiol-Heart Circ Physiol. 2000;278(5):H1679-H1685. doi:10.1152/ajpheart.2000.278.5.H1679

35. Evrard SM, Lecce L, Michelis KC, et al. Endothelial to mesenchymal transition is common in atherosclerotic lesions and is associated with plaque instability. Nat Commun. 2016;7(1):11853. doi:10.1038/ncomms11853

36. Chen PY, Qin L, Baeyens N, et al. Endothelial-to-mesenchymal transition drives atherosclerosis progression. J Clin Invest. 2015;125(12):4514-4528. doi:10.1172/JCI82719 


\section{FIGURE LEGENDS:}

Figure 1. eNOS-S1176A phospho-impaired mutant mice display exacerbated aortic lesion formation upon 12wk-Western Diet challenge. (A) No obvious weight differences were seen between the compared genotypes in either pre- or post-Western Diet. (B) En face staining for Oil Red $\mathrm{O}$ in aortas from eNOS mutant mice indicate significant lesion formation throughout the aorta in the S1176A compared to S1176D mutant mice. Quantified in (C-E). ( $\left.{ }^{\star \star \star} p<0.005 ;{ }^{* \star} p<0.01, n=6+\right)$

Figure2. Increased lipid deposition, necrotic core formation, and cellular apoptosis in phospho-impaired eNOS-S1176A mutant mice. (A) Oil Red O staining of the aortic root 12 weeks post-Western Diet challenge. Quantified in (B). Trichrome staining of the aortic root 12 weeks post-Western Diet, quantified in (D). TUNEL staining of the aortic root 12-weeks post-Western Diet, quantified in (F). ${ }^{\star \star \star \star} p<0.0001, n=3+$

Figure3. Increased CD68 and VCAM1 expression in phospho-deficient eNOSS1176A mutant mice. (A) Immunostaining of the aortic root shows enhanced expression of (A) CD68 and (B) VCAM1 within atherosclerotic lesions. Quantified in (C) and (D).

Figure 4. Gene expression analyses of whole aorta before and after 4-week Western Diet feeding. Gene expression profiles were obtained using the NanoString Immune Profile platform on whole aorta samples. (A) Heatmap of relative expression data illustrating unsupervised hierarchical gene clustering between comparison groups. Horizontal Columns: individual genes; Vertical Columns: individual mouse aorta samples. WD: Western Diet ( $<<0.01,91$ genes) (B) Pathway analyses identified several canonical pathways and (C) upstream regulators.

Figure 5. Gene expression profiles of Western Diet fed eNOS mutants. Gene expression profiles were obtained using the NanoString Immune Profile platform on whole aorta samples. (A) Heatmap of relative expression data with preferential focus on postWestern Diet feeding. Horizontal Columns: individual genes; Vertical Columns: individual mouse aorta samples. WD: Western Diet ( $p<0.01,28$ genes). (B) Pathway analyses identifies several canonical pathways and (C) predicted upstream activated regulators.

Figure 6. A 'loss-of-function' mutation together with 4-week Western Diet feeding leads to a combinatorial increase in a select cohort of interferon-associated genes. 
Gene expression profiles were obtained using the NanoString Immune Profile platform on whole aorta samples. (A) Heatmap of relative expression data with preferential focus on genes differentially upregulated in the 'SA + WD' group. Horizontal Columns: individual genes; Vertical Columns: individual mouse aorta samples. WD: Western Diet $(p<0.05$, 59 genes). (B) Pathway analyses identifies several canonical pathways and predicted upstream activated regulators.

Figure 7: TNF \& IFNy promote atherogenesis in the absence of bioavailable NO. Predicted upstream regulator pathways derived from IPA were studied to derive the networks shown above. The gene expression dataset specific to this study was superimposed on these canonical pathways to look for agreement between real data and prediction. Most of the upregulated genes in our list was also predicted by IPA to be under the regulation of either an activated TNF or IFNY.

Supp. Fig. 1. Increased lipid deposition and cellular apoptosis in phospho-impaired eNOS-S1176A mutant mice. (A) Oil Red O staining of the brachiocephalic artery 12 weeks post-Western Diet challenge. Lesion size and ORO quantified in (B) and (C). (D) TUNEL staining of the aortic root 12 -weeks post-Western Diet, quantified in (E). ${ }^{\star \star} p<$ $0.01,{ }^{*} p<0.05, n=3+$

Supp. Fig. 2. PCA plots of aorta samples. 3-dimensional PCA plot showing the samples clustering according to their groups. The PCA is based on 91 genes that are most variable between the groups in a multi-group comparison, for which the $p$-value is $\leq 0.01$. 


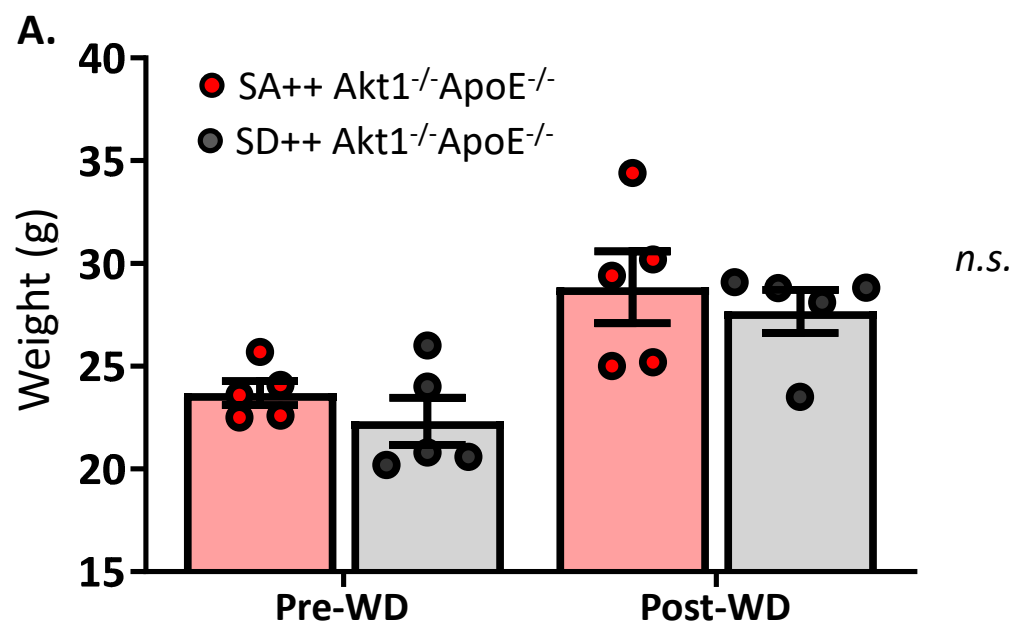

(12wk WD)

B.

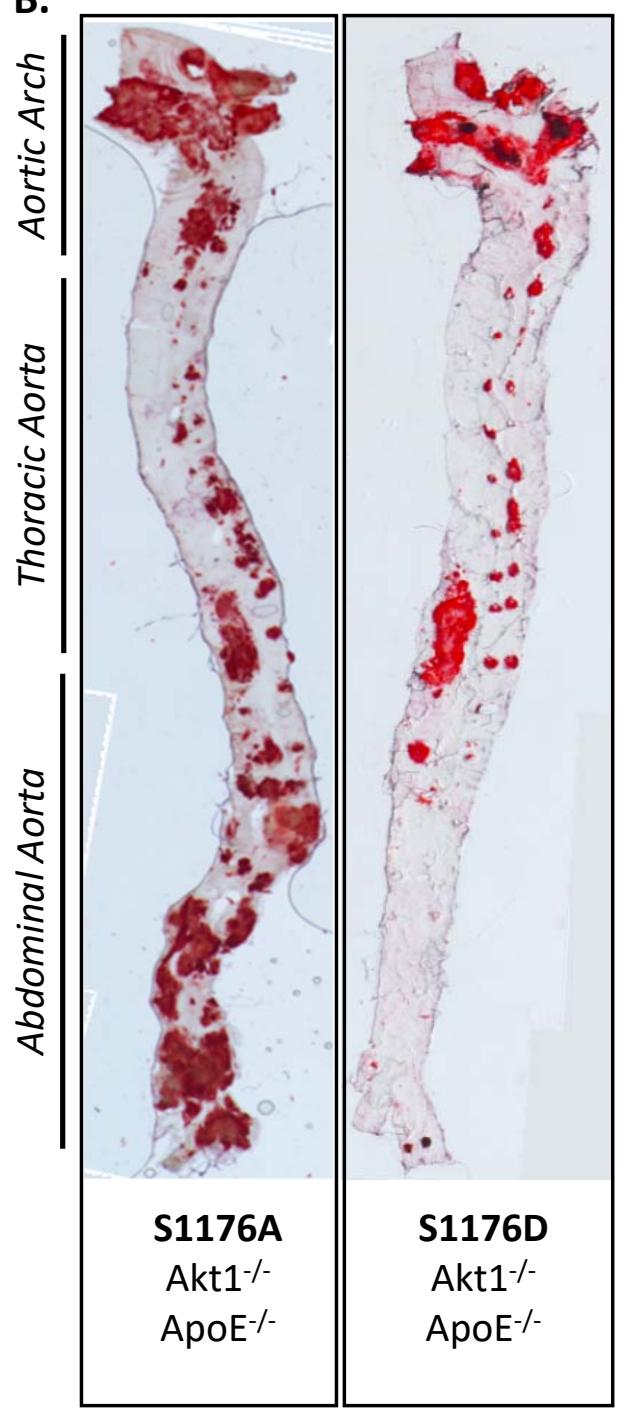

C.

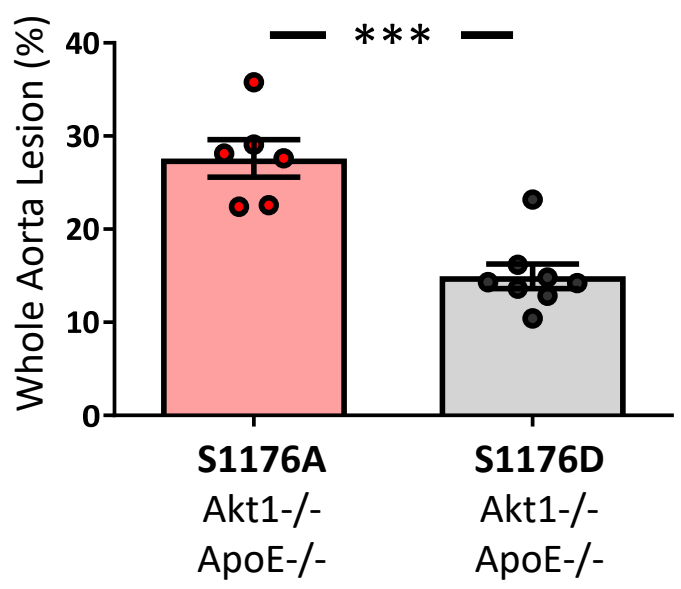

E.

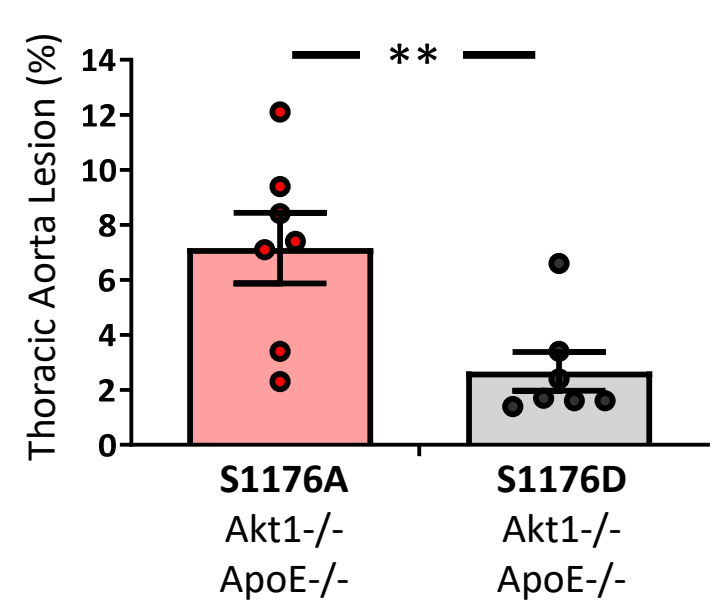

D.

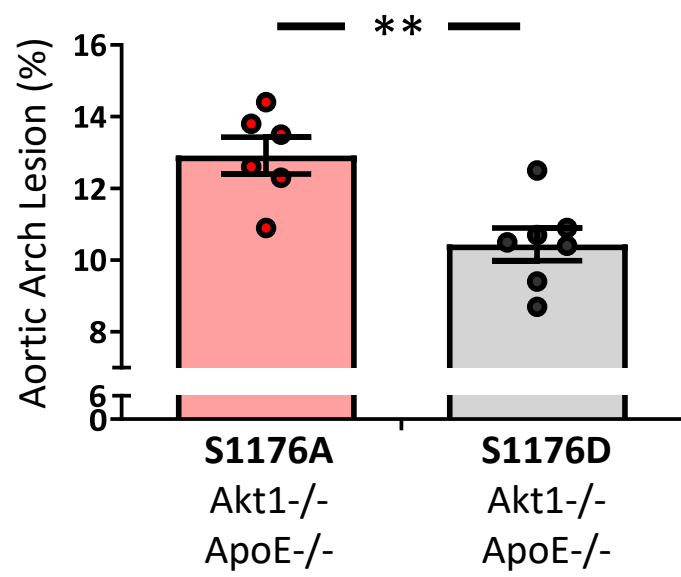

F.

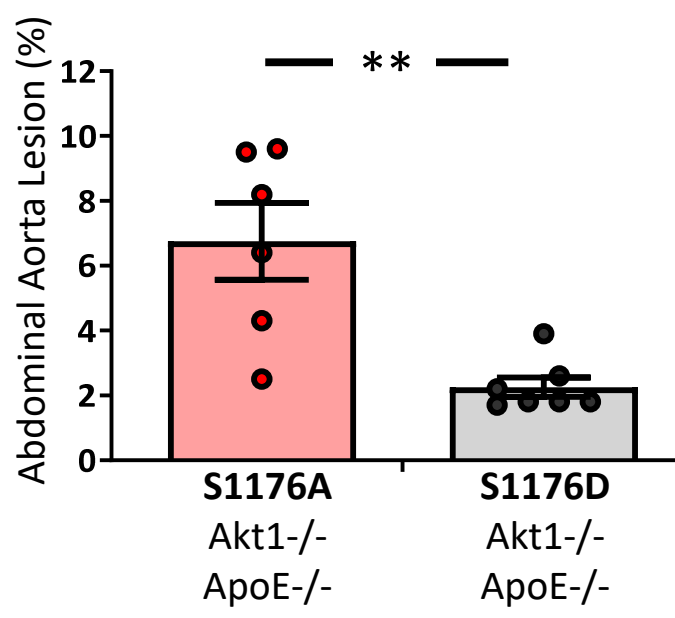

Figure 1. eNOS-S1176A phospho-impaired mutant mice display exacerbated aortic lesion formation upon 12wk-Western Diet challenge. (A) No obvious weight differences were seen between the compared genotypes in either pre- or post-Western Diet. (B) En face staining for Oil Red O in aortas from eNOS mutant mice indicate significant lesion formation throughout the aorta in the S1176A compared to S1176D mutant mice. Quantified in (C-E). 

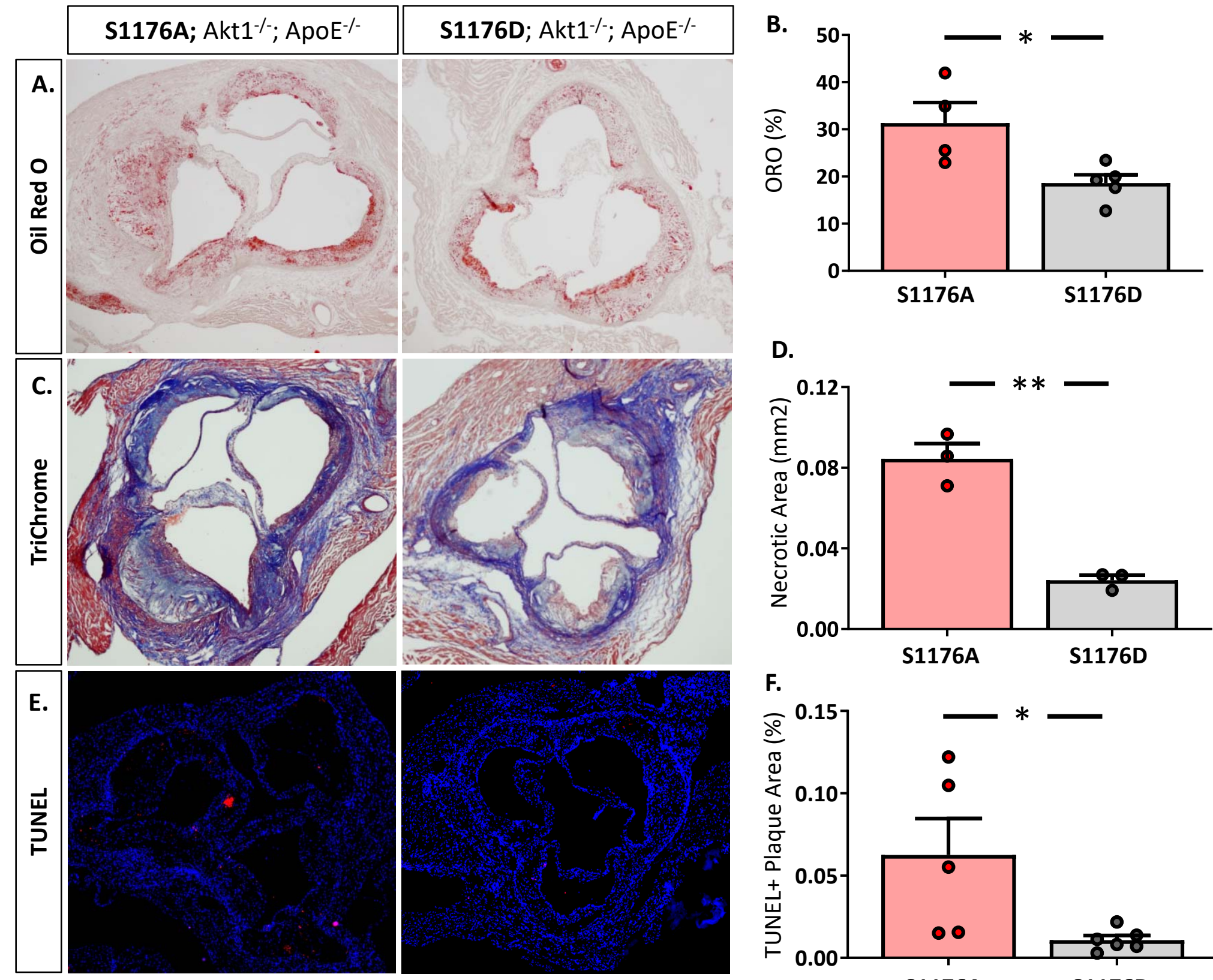

D.
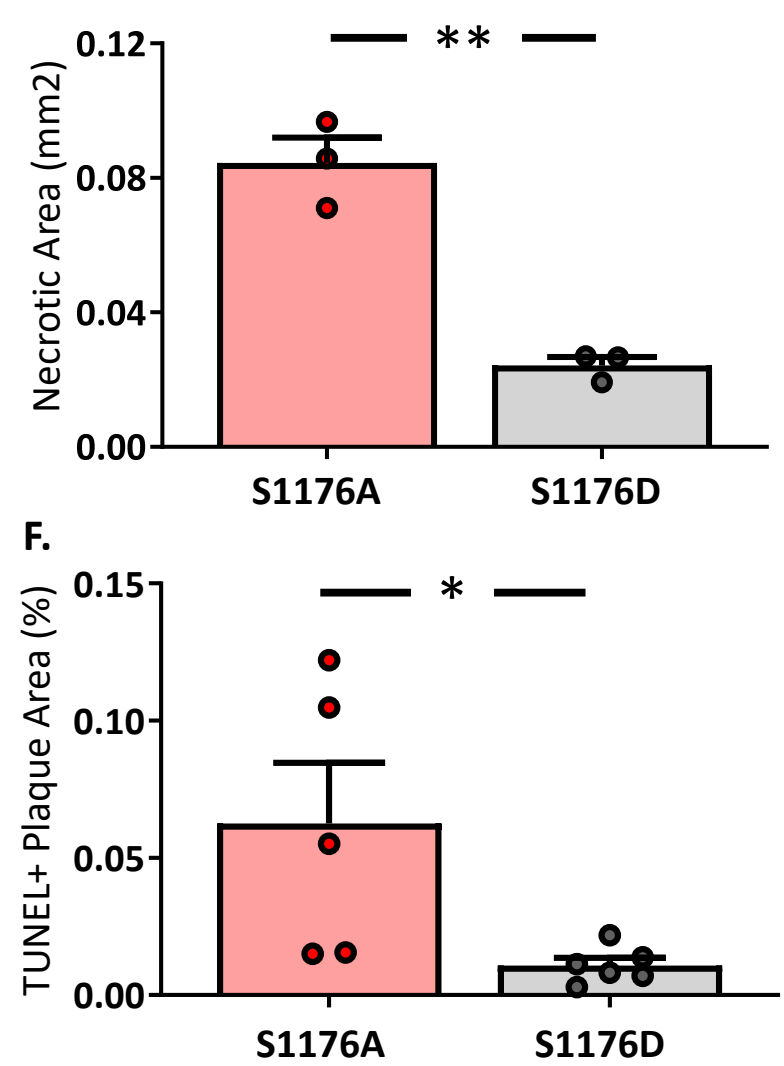

Figure2. Increased lipid deposition, necrotic core formation, and cellular apoptosis in phospho-impaired eNOS-S1176A mutant mice. (A) Oil Red O staining of the aortic root 12 weeks post-Western Diet challenge. Quantified in (B). Trichrome staining of the aortic root 12 weeks post-Western Diet, quantified in (D). TUNEL staining of the aortic root 12-weeks post-Western Diet, quantified in (F). ${ }^{* * * *} p<0.0001, n=3+$ 

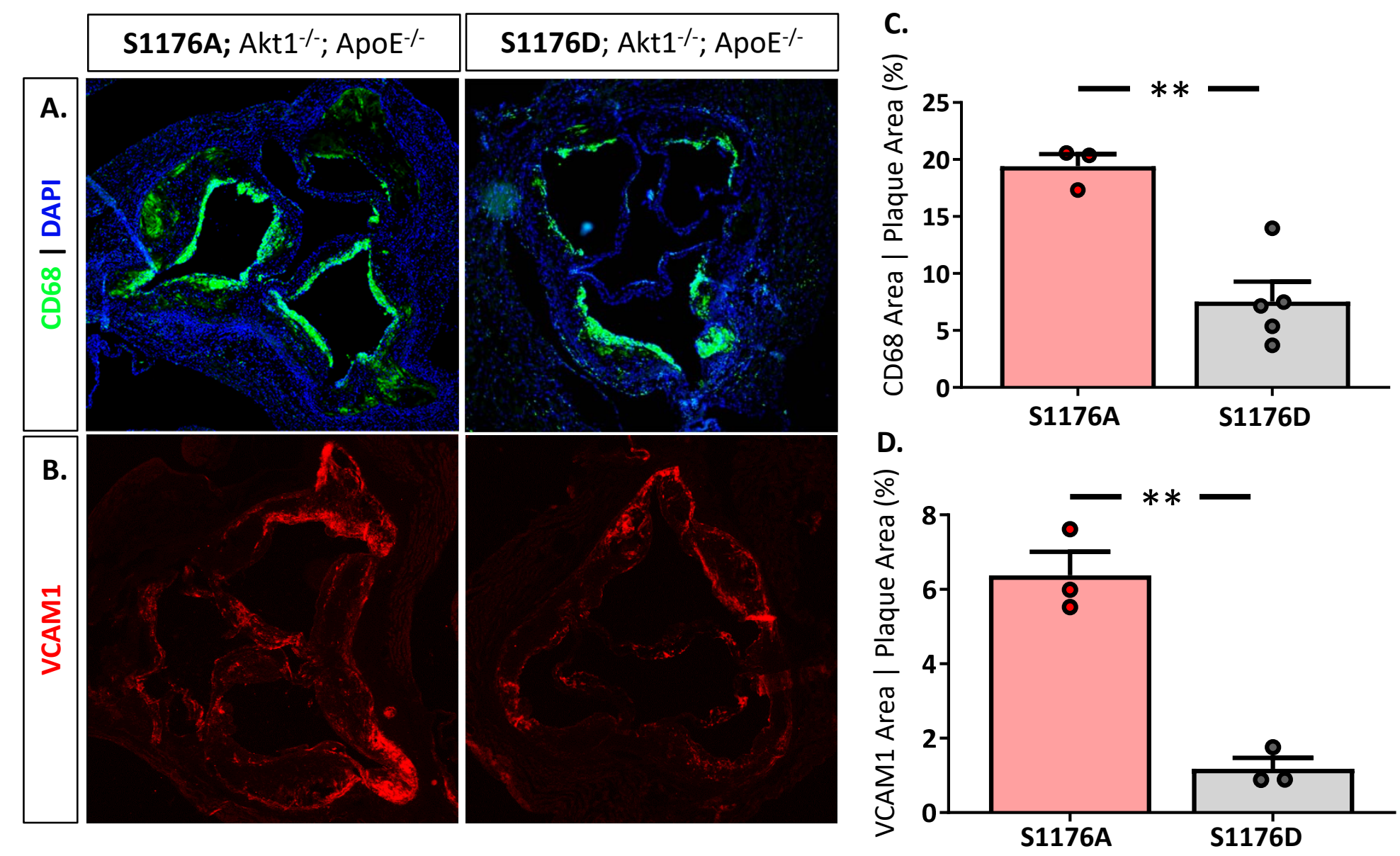

Figure3. Increased CD68 and VCAM1 expression in phospho-deficient eNOS-S1176A mutant mice. (A) Immunostaining of the aortic root shows enhanced expression of (A) CD68 and (B) VCAM1 within atherosclerotic lesions. Quantified in (C) and (D). 

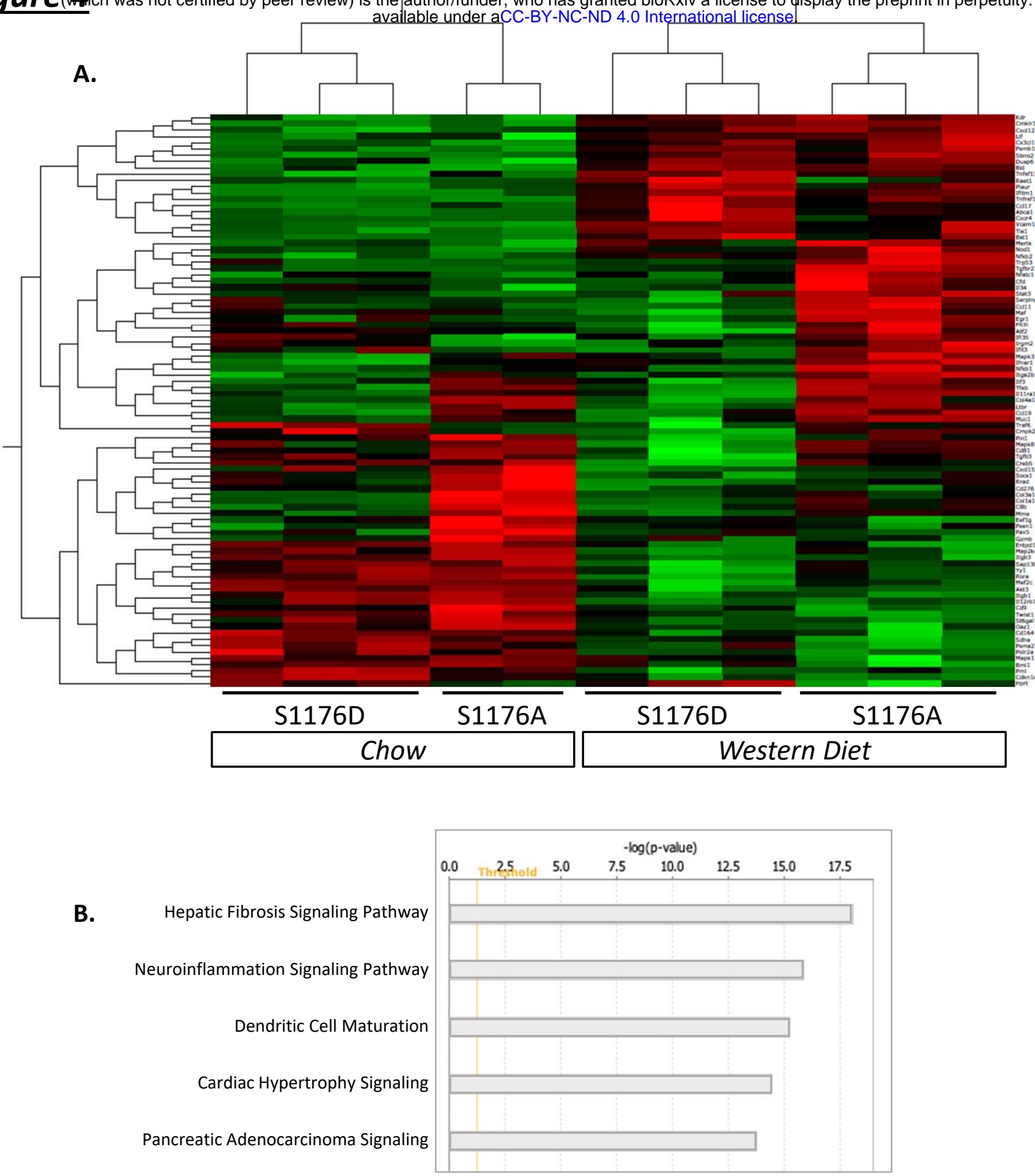

\begin{tabular}{|c|c|c|}
\hline Upstream Regulator & Molecule Type & p-value \\
\hline lipopolysaccharide & chemical drug & $1.43 \mathrm{E}-33$ \\
\hline TNF & cytokine & $4.22 \mathrm{E}-29$ \\
\hline dexamethasone & chemical drug & $7.98 \mathrm{E}-26$ \\
\hline STAT3 & transcription regulator & $1.07 \mathrm{E}-25$ \\
\hline IFNg & cytokine & $6.11 \mathrm{E}-25$ \\
\hline
\end{tabular}

Figure 4. Gene expression analyses of whole aorta before and after 4-week Western Diet feeding. Gene expression profiles were obtained using the NanoString Immune Profile platform on whole aorta samples. (A) Heatmap of relative expression data illustrating unsupervised hierarchical gene clustering between comparison groups. Horizontal Columns: individual genes; Vertical Columns: individual mouse aorta samples. WD: Western Diet ( $<<0.01,91$ genes) (B) Pathway analyses identified several canonical pathways and $(\mathbf{C})$ upstream regulators. 

available under aCC-BY-NC-ND 4.0 International license.

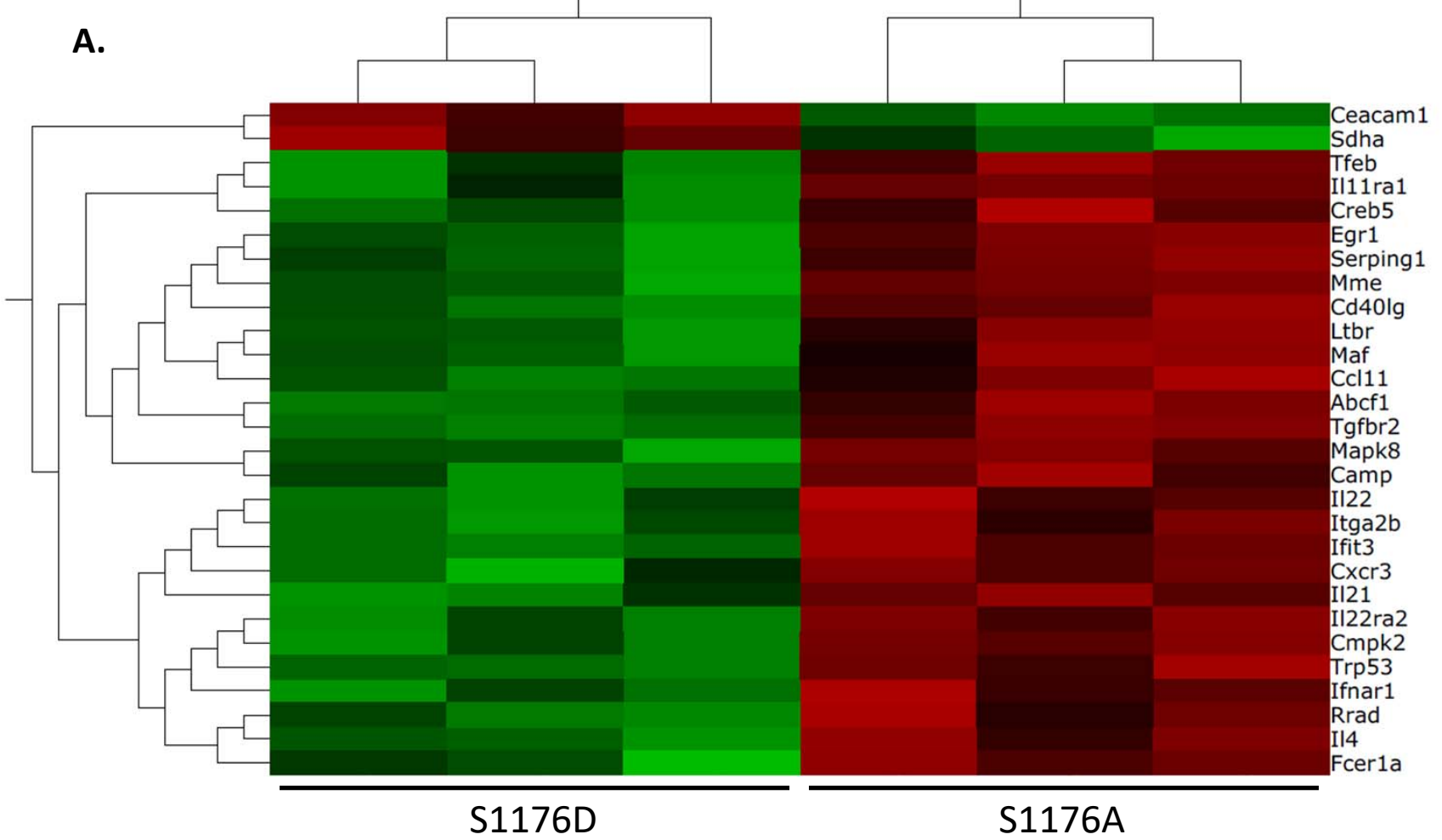

B.

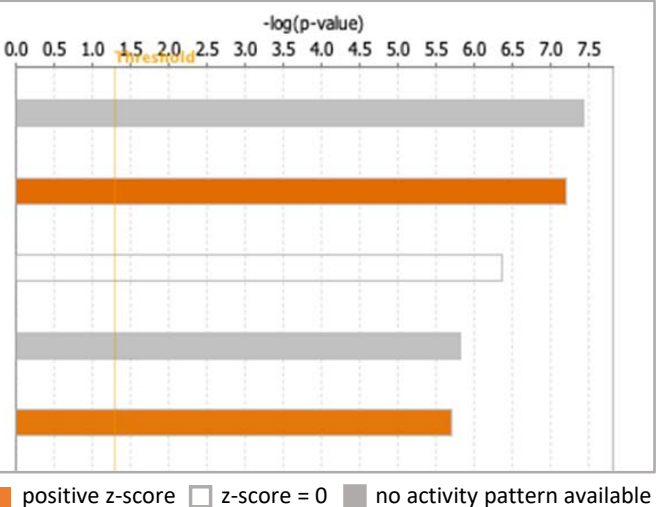

C.

\begin{tabular}{|c|c|c|c|c|}
\hline Upstream Regulator & Molecule Type & Predicted Activation State & Activation z-score & p-value \\
\hline TCR & complex & Activated & 2.072 & $4.15 \mathrm{E}-10$ \\
\hline NFkB & complex & Activated & 2.412 & 8.63E-06 \\
\hline TNF & cytokine & Activated & 2.214 & $9.60 E-08$ \\
\hline IL1b & cytokine & Activated & 2.609 & $1.80 \mathrm{E}-05$ \\
\hline CD28 & transmembrane receptor & Activated & 2.186 & $3.51 \mathrm{E}-06$ \\
\hline PLX5622 & chemical drug & Activated & 2.000 & 2.27E-06 \\
\hline ionomycin & chemical reagent & Activated & 2.598 & $2.96 \mathrm{E}-09$ \\
\hline lipopolysaccharide & chemical drug & Activated & 2.406 & $1.52 \mathrm{E}-10$ \\
\hline tetradecanoylphorbol acetate & chemical drug & Activated & 2.132 & $5.67 \mathrm{E}-09$ \\
\hline prednisolone & chemical drug & Inhibited & -2.000 & $2.16 \mathrm{E}-04$ \\
\hline dexamethasone & chemical drug & Inhibited & -2.235 & 2.39E-12 \\
\hline LY294002 & chemical - kinase inhibitor & Inhibited & -2.000 & $3.72 \mathrm{E}-05$ \\
\hline SB203580 & chemical - kinase inhibitor & Inhibited & -2.153 & $6.19 \mathrm{E}-08$ \\
\hline filgrastim & biologic drug & Inhibited & -2.216 & $1.58 \mathrm{E}-04$ \\
\hline
\end{tabular}

Figure 5. Gene expression profiles of Western Diet fed eNOS mutants. Gene expression profiles were obtained using the NanoString Immune Profile platform on whole aorta samples. (A) Heatmap of relative expression data with preferential focus on post-Western Diet feeding. Horizontal Columns: individual genes; Vertical Columns: individual mouse aorta samples. WD: Western Diet ( $p<0.01,28$ genes). (B) Pathway analyses identifies several canonical pathways and (C) predicted upstream activated regulators. 


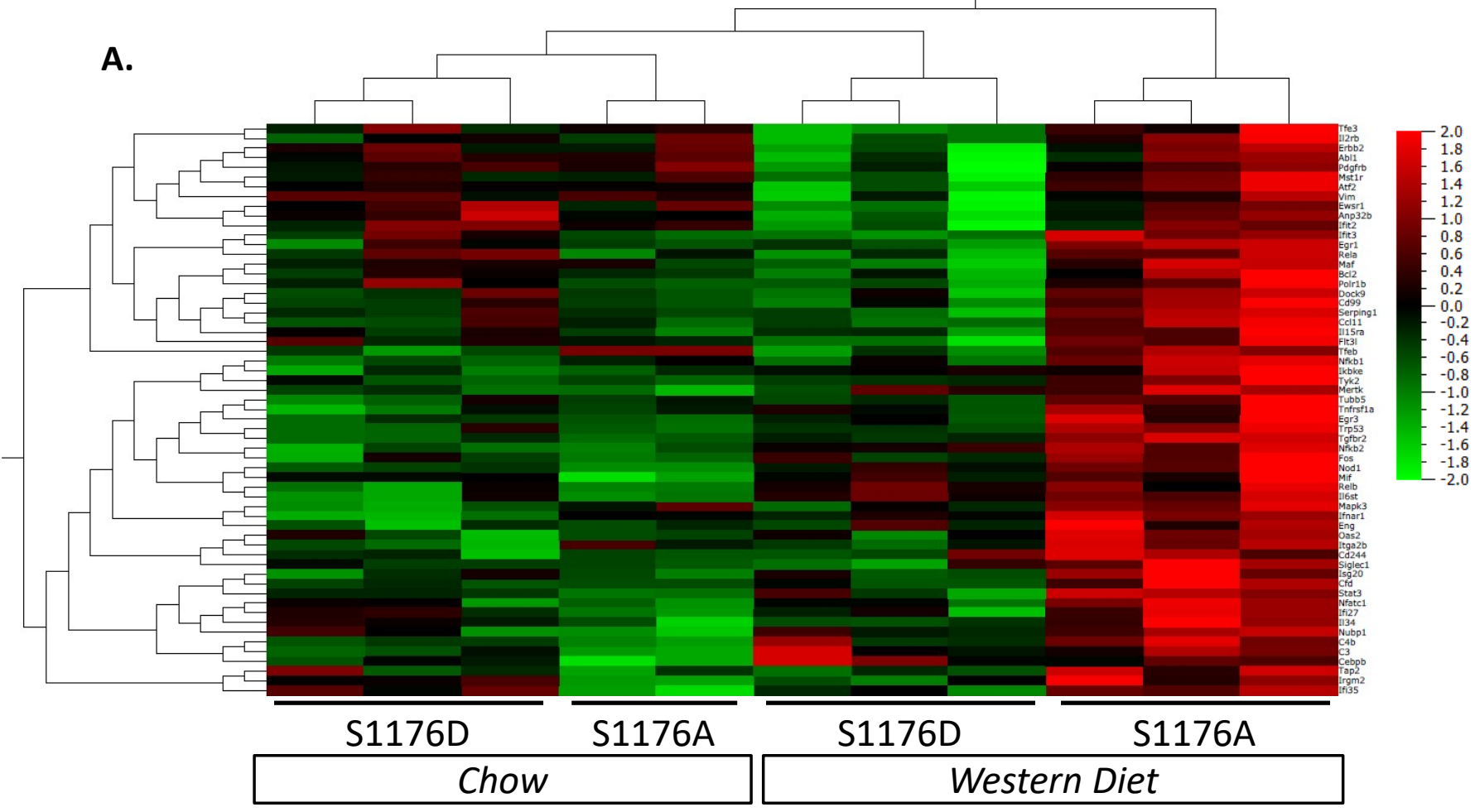

B.

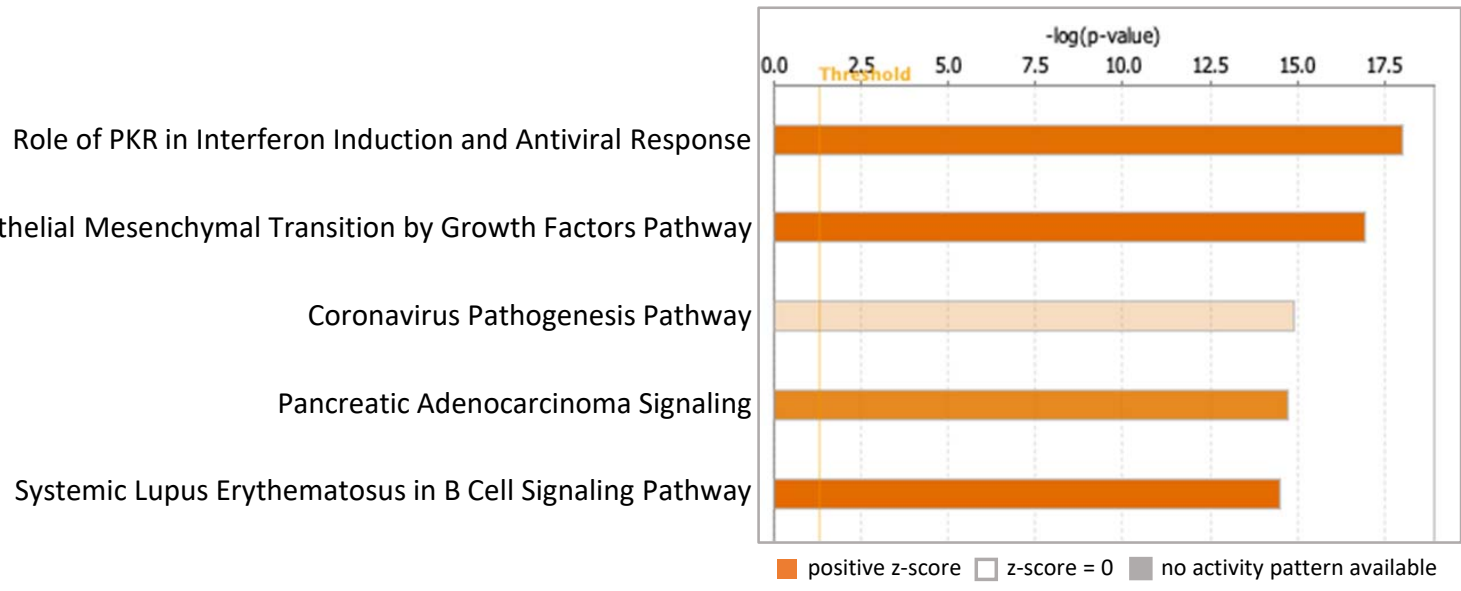

C.

\begin{tabular}{|c|c|c|c|c|}
\hline Upstream Regulator & Molecule Type & Predicted Activation State & Activation z-score & p-value \\
\hline lipopolysaccharide & chemical drug & Activated & 3.517 & $6.44 \mathrm{E}-21$ \\
\hline RELA & transcription regulator & Activated & 3.508 & $3.07 E-20$ \\
\hline tetradecanoylphorbol acetate & chemical drug & Activated & 3.426 & $2.71 E-12$ \\
\hline IFNg & cytokine & Activated & 3.410 & 1.25E-21 \\
\hline TNF & cytokine & Activated & 3.303 & $3.37 E-16$ \\
\hline TLR4 & transmembrane receptor & Activated & 3.250 & $1.86 \mathrm{E}-14$ \\
\hline IL2 & cytokine & Activated & 3.239 & $2.54 \mathrm{E}-10$ \\
\hline AGT & growth factor & Activated & 3.218 & $1.90 \mathrm{E}-11$ \\
\hline HGF & growth factor & Activated & 3.135 & $1.14 \mathrm{E}-11$ \\
\hline Ige & complex & Activated & 3.132 & $6.55 \mathrm{E}-09$ \\
\hline
\end{tabular}

Figure 6. A 'loss-of-function' mutation together with 4-week Western Diet feeding leads to a combinatorial increase in a select cohort of interferon-associated genes. Gene expression profiles were obtained using the NanoString Immune Profile platform on whole aorta samples. (A) Heatmap of relative expression data with preferential focus on genes differentially upregulated in the 'SA + WD' group. Horizontal Columns: individual genes; Vertical Columns: individual mouse aorta samples. WD: Western Diet ( $p<0.05,59$ genes). (B) Pathway analyses identifies several canonical pathways and predicted upstream activated regulators. 
bipxiv preprint doi: https://doi.org/10.1101/2022.01.13.476234; this version posted January 16, 2022. The copyright holder for this preprint Figure (which was not certified by peer review) is the author/funder, who has granted bioRxiv a license to display the preprint in perpetuity. It is made available under aCC-BY-NC-ND 4.0 International license.

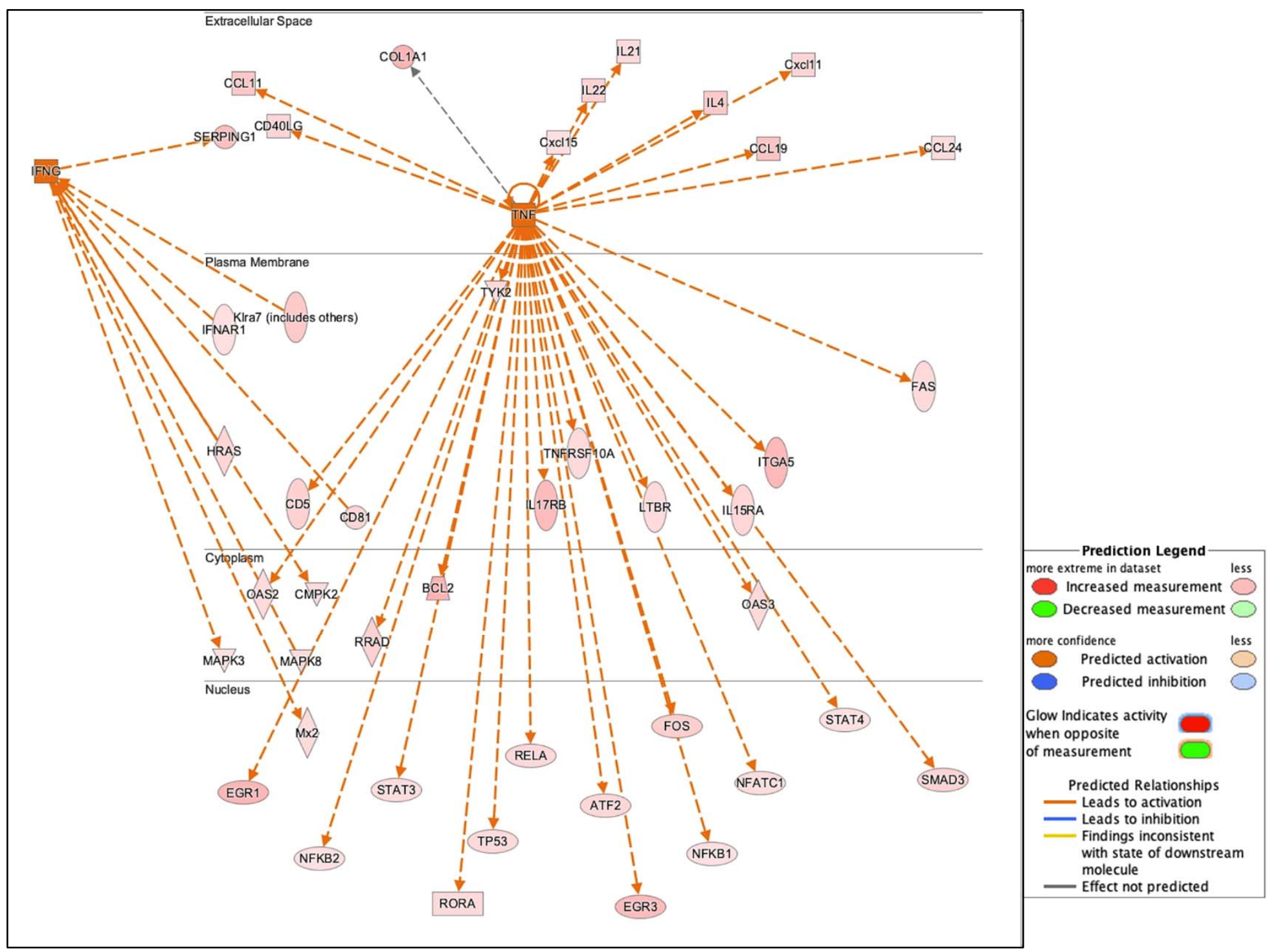

Figure 7: TNF \& IFNY promote atherogenesis in the absence of bioavailable NO. Predicted upstream regulator pathways derived from IPA were studied to derive the networks shown above. The gene expression dataset specific to this study was super-imposed on these canonical pathways to look for agreement between real data and prediction. Most of the upregulated genes in our list was also predicted by IPA to be under the regulation of either an activated TNF or IFN $\gamma$. 